\title{
Erratum to: High response rate and improved exercise capacity and quality of life with a new regimen of darbepoetin alfa with or without filgrastim in lower-risk myelodysplastic syndromes: a phase II study by the GFM
}

\author{
C. Kelaidi • O. Beyne-Rauzy • T. Braun • R. Sapena • \\ P. Cougoul • L. Adès $\cdot$ F. Pillard $\cdot$ C. Lamberto • \\ J. C. Charniot • A. Guerci • B. Choufi • A. Stamatoullas • \\ B. Slama • B. De Renzis • S. Ame • G. Damaj • F. Boyer • \\ M. P. Chaury • L. Legros • S. Cheze • A. Testu • E. Gyan • \\ M. C. Béné $\cdot$ C. Rose $\cdot$ F. Dreyfus $\cdot$ P. Fenaux
}

Published online: 6 March 2013

(C) Springer-Verlag Berlin Heidelberg 2013

\section{Erratum to: Ann Hematol \\ DOI 10.1007/s00277-013-1686-4}

The original version of this article inadvertently contained a mistake. The name of the 8th author has been misspelled as Christine LAMBERT while the correct name is Christine LAMBERTO.

The online version of the original article can be found at http://dx.doi.org/ 10.1007/s00277-013-1686-4.

C. Kelaidi · O. Beyne-Rauzy • T. Braun · R. Sapena $\cdot$ P. Cougoul $\cdot$

L. Adès $\cdot$ F. Pillard $\cdot$ C. Lamberto $\cdot$ J. C. Charniot $\cdot$ A. Guerci $\cdot$

B. Choufi - A. Stamatoullas $\cdot$ B. Slama $\cdot$ B. De Renzis $\cdot$ S. Ame

G. Damaj $\cdot$ F. Boyer $\cdot$ M. P. Chaury $\cdot$ L. Legros $\cdot$ S. Cheze $\cdot$

A. Testu $\cdot$ E. Gyan $\cdot$ M. C. Béné $\cdot$ C. Rose $\cdot$ F. Dreyfus $\cdot$

P. Fenaux $(\square)$

GFM Service d'Hématogie Clinique, Hôpital Avicenne,

Assistance Publique des Hôpitaux de Paris, Université Paris 13,

125 rue de Stalingrad,

Bobigny 93009, France

e-mail: pierre.fenaux@avc.aphp.fr 